\title{
LINEARLY ORDERED COLLECTIONS AND PARACOMPACTNESS
}

\author{
J. E. VAUGHAN
}

1. Introduction. The purpose of this paper is to characterize paracompactness by conditions which are formally weaker than some considered by Michael [3], [4], [5], and which are related to some considered by Tamano [7], [8], and Katuta [2]. In addition some consequences of these concepts are derived. The main result is the following

TheOREM 1. Let $X$ be a regular space. The following are equivalent

(a) $X$ is paracompact.

(b) Every open cover of $X$ has an open refinement which is linearly locally finite.

(c) Every open cover of $X$ has an open refinement which is linearly closure-preserving.

(d) Every open cover of $X$ has an open refinement which is linearly cushioned in it.

Let us now define all of the terms which are mentioned in Theorem 1. Let $\mathcal{u}$ and $v$ be collections of subsets of a topological space. A collection $\mathcal{u}$ endowed with a linear $(=$ total) order is said to be linearly locally finite with respect to $\leqq$ provided that every majorized subcollection (that is, every subcollection of $u$ having an upper bound with respect to $\leqq$ ) is locally finite. This definition is equivalent to that used by H. Tamano in [7] where he proved that (a) and (b) in Theorem 1 are equivalent in completely regular spaces. A collection $u$ endowed with a linear order is said to be linearly closure-preserving with respect to $\leqq$ provided that every majorized subcollection of $\mathfrak{u}$ is closure-preserving. In order to define the term mentioned in (d), we first restate a definition given in [5]. A collection $u$ is said to be cushioned in a collection $v$ with cushion map $f: U \rightarrow \mathcal{U}$ provided for every subcollection $\mathfrak{u}^{\prime}$ of $u$ we have $\operatorname{cl}\left(\cup \mathcal{u}^{\prime}\right) \subset \cup f\left(\mathcal{u}^{\prime}\right)$. We say that a collection $\mathfrak{U}$ endowed with a linear order is linearly cushioned in a collection $v$ with cushion map $f: \mathcal{u} \rightarrow \mathcal{V}$ provided for every majorized subcollection $\mathfrak{u}^{\prime}$ of $\mathfrak{u}$ we have $\operatorname{cl}\left(\cup \mathcal{u}^{\prime}\right) \subset \bigcup f\left(\mathcal{u}^{\prime}\right)$. We will omit explicit mention of the cushion map if no confusion will result.

The above definitions of linearly closure-preserving and "linearly cushioned" differ from those given by $\mathrm{H}$. Tamano in [8] where he 1968.

Presented to the Society August 30,1968; received by the editors September 9 
required the linear order to be a well-order. In that paper he proved (using his definitions) that (a), (c), and (d) of Theorem 1 are equivalent in a completely regular space. The proof of Theorem 1 is given in $\$ 2$, and is different from Tamano's proof because he made use of the Stone-Čech compactification of completely regular spaces. This method, of course, is not available for arbitrary regular spaces.

In $\S 3$ we consider the relationship of the "linear concepts" to the concepts considered by Michael. In $\$ 4$ we consider the consequences of replacing the word "majorized" by the word "bounded" in the above definitions. In $\$ 5$ we discuss the concept of order local finiteness in the sense of Katuta, and give an analogue of Theorem 1.

2. Proof of Theorem 1. We begin with a key set-theoretic lemma.

LEMMA 1. Every set with a linear order $\leqq$ can be given a well-ordering $\leqq \leqq$ such that every $\leqq \leqq$ majorized set is also $\leqq$ majorized.

Proof. Let $\leqq$ be a linear order on a set $X$. Let $B$ be a well-ordered cofinal subset of $X$. If $B$ has a largest element, then any well-order on $X$ will work. We assume $B$ has no largest element with respect to $\leqq$. For every $b \in B$ define $A_{b}=\{x \in X: x<b\}$, and

$$
D_{b}=A_{b}-\cup\left\{A_{p}: p<b \text { and } p \in B\right\} .
$$

Notice that $\left\{D_{b}: b \in B\right\}$ is a partition of $X$. Now let $\leqq_{b}$ be any wellorder on $D_{b}$ for every $b \in B$, and define an order $\leqq \leqq$ on $X$ as follows. Let $x, y \in X$, then $x \leqq \leqq y$ if and only if

(1) there exists $b \in B$ such that $x, y \in D_{b}$ and $x \leqq_{b} y$, or

(2) there exist $b, c \in B$ such that $x \in D_{b}, y \in D_{c}$ and $b<c$.

It is routine to check that $\leqq \leqq$ is a well-order for $X$ having the desired property.

The author does not know if every linearly closure-preserving open cover of a topological space has a closure-preserving refinement. For the other kinds of collections, however, one can prove the following

Lemma 2. Let $X$ be a topological space. Every open cover $\mathcal{u}$ of $X$ which is linearly locally finite (resp. linearly cushioned in $v$ ) has a refinement -not necessarily open-which is locally finite (resp. cushioned in $\mathrm{U})$.

Proof. We prove the second case. Let $u$ be linearly cushioned in $\mathcal{U}$ with respect to $\leqq$. Let $\leqq \leqq$ be a well-order on $\mathcal{u}$ such that every $\leqq \leqq$ majorized subset of $\mathcal{u}$ is $\leqq$ majorized. Clearly, $\mathcal{u}$ is linearly cushioned in $V$ with respect to $\leqq \leqq$ and with the same cushion map $f: u \rightarrow v$. Define $H_{U}=U-U\{V \in \mathcal{u}: V<<U\}$ for every $U \in \mathfrak{u}$. Let $\mathfrak{H C}=\left\{H_{U}: U \in \mathcal{U}\right\}$, then $\mathfrak{H C}$ is a refinement of $\mathcal{U}$ and is cushioned in $v$ 
with cushion map $g: \mathfrak{F} \rightarrow \mathcal{U}$ defined by $g\left(H_{U}\right)=f(U)$. To see that $\mathfrak{H C}$ covers $X$, let $x \in X$, and let $U$ be the first element of $u$ containing $x$, then $x \in H_{U}$. To see that $\mathfrak{H}$ is cushioned in $\mathcal{V}$, let $\mathfrak{H}^{\prime}$ be any subcollection of $\mathfrak{H}$, and let $p$ be in $\operatorname{cl}\left(\cup \mathcal{F}^{\prime}\right)$. Since $\mathcal{U}$ is an open cover of $X$, there exists $U \in \mathcal{u}$ such that $p \in U$. Then $U$ is an open set which misses $H_{V}$ for all $V>>U$. Set $\mathcal{F}^{\prime \prime}=\left\{H_{V}: V \leqq \leqq U\right.$, and note that $p \in \operatorname{cl}\left(U_{\mathcal{F}^{\prime \prime}}\right)$. The subcollection $\mathcal{u}^{\prime \prime}=\left\{V \in \mathcal{U}: H_{V} \in \mathcal{F}^{\prime \prime}\right\}$ is majorized by $U$ hence $\operatorname{cl}\left(U \mathcal{U}^{\prime \prime}\right) \subset \cup f\left(\mathcal{U}^{\prime \prime}\right)=U g\left(\mathcal{F}^{\prime \prime}\right)$. It follows that $p \in U g\left(\mathcal{H C}^{\prime}\right)$, and this implies that $\operatorname{cl}\left(\cup_{\mathcal{F}^{\prime}}\right) \subset U_{g}\left(\mathcal{F C}^{\prime}\right)$.

Proof of TheOREM 1. The implications $(\mathrm{a}) \Rightarrow(\mathrm{b}) \Rightarrow(\mathrm{c}) \Rightarrow(\mathrm{d})$ are obvious. It remains to prove $(d) \Rightarrow(a)$. By Theorem 1.1 of [5], it suffices to show that every open cover has a refinement which is cushioned in it, but this follows immediately from Lemma 2.

3. Relations of linearly ordered collections to some other properties. It is clear that every $\sigma$-locally finite collection is a linearly locally finite collection. Conversely, we have the following obvious

PROPOSITION 1. Let $u$ be a collection which is linearly locally finite in a space $X$.

(a) If $\mathfrak{u}$ has a largest element, then $\mathfrak{u}$ is locally finite.

(b) If $\mathfrak{u}$ has a countable cofinal subset, then $\mathcal{u}$ is $\sigma$-locally finite.

(c) If $\mathcal{u}$ does not have a countable cofinal subset, then $\mathfrak{u}$ is point-finite (i.e., every point in $X$ is a member of only finitely many elements of $\mathfrak{u}$ ).

Remark. Proposition 1 (a) and (b) remain true if everywhere the words "locally finite" are replaced by "closure-preserving," or "cushioned in $0 . "$

Proposition 2. In a space $X$ which satisfies the first axiom of countability, every collection which is linearly locally finite is $\sigma$-locally finite.

PROOF. Let $u$ be linearly locally finite with respect to a linear order $\leqq$. Assume $\mathcal{u}$ is not $\sigma$-locally finite; so $\mathcal{U}$ does not have a countable cofinal subset. Since $\mathcal{U}$ is not locally finite there exists $x \in X$ such that every neighborhood of $x$ intersects infinitely many members of $u$. Let $\left\{N_{i}(x): i=1,2, \cdots\right\}$ be a fundamental system of neighborhoods of $x$ such that $N_{i}(x) \supset N_{i+1}(x)$. Then there exists an infinite sequence $\mathfrak{u}^{\prime}=\left\{U_{i}: i=1,2, \cdots\right\}$ of distinct elements of $u$ such that $U_{i} \cap N_{i}(x) \neq \varnothing$. Since $u^{\prime}$ is not cofinal in $u$ it is majorized, and therefore locally finite. Thus, there exists $N_{i}(x)$ which intersects only finitely many elements of $\mathcal{u}^{\prime}$. But this is impossible since $N_{i}(x) \cap U_{n}$ $\neq \varnothing$ for $n \geqq i$.

REMARK. Proposition 2 remains true if everywhere the words "locally finite" are replaced by "closure-preserving" or "cushioned in 
v." The referee pointed out that a similar proof can be given to show that Proposition 2 holds in a $T_{1}$-space which is a $q$-space in the sense of E. Michael [6]. Further, one can show that in a $T_{1}$-regular $q$-space every linearly closure-preserving collection is $\sigma$-closure-preserving. It is not possible, however, to extend Proposition 2 to $q$-spaces and cushioned collections. Here is a simple example. Let $\Omega$ be the first uncountable ordinal, then $[0, \Omega]$ with the order topology is a compact Hausdorff space (hence a $q$-space). Let $v=\{[0, \Omega)\}$, and let $u$ $=\{\{p\}: p \in[0, \Omega)\}$. It is easy to see that $u$ is linearly cushioned in $\mathcal{v}$ with respect to the usual order on $[0, \Omega)$, but $\mathcal{U}$ is not $\sigma$-cushioned in $v$.

An immediate consequence of the preceding propositions is the following modification of the Nagata-Smirnov Theorem. Call a base for a topology a $\sigma$-linearly locally finite base if it is a countable union of linearly locally finite collections.

Corollary. A regular $T_{1}$-space is metrizable if and only if its topology has a $\sigma$-linearly locally finite base.

We now partially strengthen Lemma 2 for normal spaces.

Proposition 3. Let $X$ be a normal space. Every linearly locally finite open cover of $X$ has a $\sigma$-locally finite open refinement.

Proof. Let $u$ be a linearly locally finite open cover of $X$. If $\mathcal{u}$ is $\sigma$-locally finite, then there is nothing to prove. If $\mathcal{U}$ is not $\sigma$-locally finite, then by Proposition 1 it is point-finite. This means that $u$ is shrinkable, i.e., there exists an open cover $\left\{H_{U}: U \in \mathcal{U}\right\}$ of $X$ such that $\bar{H}_{U} \subset U$ for all $U \in \mathcal{u}$. Let $W_{U}=U-U\left\{\bar{H}_{V}: V<U\right\}$. Then $W=\left\{W_{U}: U \in \mathcal{U}\right\}$ is in fact a locally finite open refinement of $\mathcal{u}$.

REMARK. In light of Proposition 3 it is, perhaps, interesting to note that every $\sigma$-locally finite open cover of a normal $T_{2}$-space has a locally finite open refinement if and only if every normal $T_{2}$-space is countably paracompact. Whether every normal $T_{2}$-space is countably paracompact is a well-known unsolved problem raised by $\mathrm{C}$. $\mathrm{H}$. Dowker.

From Theorem 1 we see that if every open cover of a regular space has a linearly locally finite open refinement, then the space is paracompact. The following question arises: If every open cover of a regular space has a $\sigma$-linearly locally finite open refinement (i.e., a refinement which is a countable union of linearly locally finite open collections), then is the space paracompact? The following example shows that the answer is in the negative.

ExAmple 1. A completely regular $T_{2}$-space $X$ which is not normal (hence not paracompact), and every open cover of $X$ has a $\sigma$-linearly 
locally finite open refinement. This example is due to J. Dieudonné [1]. Let $\omega, \Omega$ be the first infinite and the first uncountable ordinals respectively. Let $X=[0, \Omega] \times[0, \omega]-(\Omega, \omega)$. For every $n<\omega$ let $\left\{U_{x, n}=[x, \Omega] \times\{n\}: x<\Omega\right\}$ be a fundamental system of neighborhoods of $(\Omega, n)$. Let $\left\{V_{x, n}=\{x\} \times[n, \omega]: n<\omega\right\}$ be a fundamental system of neighborhoods of $(x, \omega)$. All other points $(x, n)$ for $x<\Omega$, $n<\omega$ are to be isolated. Let $u$ be any open cover of $X$. For every $n<\omega$ (resp. $x<\Omega$ ) let $U_{n}=U_{x, n}$ (resp. $V_{x}=V_{x, n}$ ) be a basic open neighborhood of $(\Omega, n)$ (resp. $(x, \omega)$ ) which is contained in some element of $\mathcal{U}$. Let $V_{1}=\left\{U_{n}: n \in[0, \omega)\right\}$, and order $\mho_{1}$ by the usual order on $[0, \omega)$. Let $V_{2}=\left\{V_{x}: x \in[0, \Omega)\right\}$, and order $V_{2}$ by the usual order on $[0, \Omega)$. Let $v_{3}$ be the set of all points which are not contained in any element of $V_{1} \cup V_{2}$, and give $V_{3}$ any well-order. Clearly, $\bigcup_{i=1}^{3} V_{i}$ is an open $\sigma$-linearly locally finite refinement of $u$.

4. Majorized versus bounded. The main purpose of this section is to show that the conditions in Theorem 1 cannot be weakened by replacing the word "majorized" by "bounded (above and below)" in the definitions given in $\$ 1$. To facilitate further discussion we make the following definition: A collection $\mathcal{u}$ endowed with a linear order $\leqq$ is said to be weakly linearly locally finite with respect to $\leqq$ provided every bounded subcollection of $\mathcal{U}$ is locally finite. It is clear that every linearly locally finite collection is weakly linearly locally finite. If there is a least element for the linear order, then the converse holds. In any case we have

Proposition 4. A collection $u$ is weakly linearly locally finite if and only if $\mathfrak{u}=\mathfrak{u}_{1} \cup \mathfrak{u}_{2}$ with each of $\mathfrak{u}_{1}$ and $\mathfrak{u}_{2}$ linearly locally finite.

Proof. To show the only if part, let $U_{0} \in \mathcal{U}$ and define

$$
\mathfrak{u}_{1}=\left\{U \in \mathfrak{u}: U_{0} \leqq U\right\} .
$$

Give $\mathfrak{u}_{1}$ the induced order from $\mathfrak{u}$. Let $\mathfrak{u}_{2}=\mathfrak{u}-\mathfrak{u}_{1}$, and give $\mathfrak{u}_{2}$ the order induced from the inverse of the original order relation on $\mathcal{u}$. Then $u_{1}$ and $\mathfrak{u}_{2}$ are linearly locally finite.

To prove the if part, let $\mathfrak{U}_{i}$ be linearly locally finite with respect to $\leqq_{i}$ for $i=1,2$, and let $\mathfrak{u}_{2}^{\prime \prime}=\mathfrak{u}_{2}-\mathfrak{u}_{1}$. Define a linear order on $\mathfrak{u}$ as follows. If $U, U^{\prime} \in \mathcal{U}$, then $U \leqq U^{\prime}$ if and only if (1) $U \in \mathcal{u}_{2}^{\prime \prime}$ and $U^{\prime} \in \mathfrak{u}_{1},(2) U, U^{\prime} \in \mathcal{u}_{1}$ and $U \leqq{ }_{1} U^{\prime}$, or (3) $U, U^{\prime} \in \mathcal{u}_{2}^{\prime \prime}$ and $U^{\prime} \leqq{ }_{2} U$. Then $\mathcal{U}$ is weakly linearly locally finite with respect to $\leqq$.

The space $X$ of Example 1 is a nonparacompact space in which every open cover has a weakly linearly locally finite open refinement. This is easy to see because $V_{3}$ in that example is actually locally 
finite. If we let $V_{1}^{\prime}=V_{3} \cup V_{1}$, and order $\mathcal{V}_{1}^{\prime}$ in such a way that every element of $V_{3}$ precedes every element of $V_{1}$, then $V_{1}^{\prime}$ is also linearly locally finite. By Proposition 4, $v=\mho_{1}^{\prime} \cup \mho_{2}$ is weakly linearly locally finite.

REMARK. In a similar manner one may define "weakly linearly closure-preserving" and "weakly linearly cushioned in $v$ ". The obvious analogues of Proposition 4 still hold.

5. Order local finiteness. Y. Katuta [2] called a collection $u$ endowed with a linear order $\leqq$ order locally finite with respect to $\leqq$ provided for every $U \in \mathcal{U}$ the collection $\{V \in \mathcal{U}: V \leqq U\}$ is locally finite at each point of $U$. He also proved [2, Lemma 2, p. 616] that a regular space is paracompact if and only if every open cover has an open order locally finite refinement. This is a stronger result than (b) $\Rightarrow$ (a) in Theorem 1, and his proof did not use well-ordering. It is natural to ask if all of Theorem 1 can be similarly strengthened. The answer is in the negative for the following extension of Katuta's concept. We say that a collection $\mathcal{U}$ endowed with a linear order $\leqq$ is order closure-preserving provided for every $U \in \mathcal{U}$ and every $x \in U$ and every subcollection $\mathcal{u}^{\prime} \subset\{V \in \mathcal{U}: V \leqq U\}$, if $x \in \operatorname{cl}\left(U \mathcal{u}^{\prime}\right)$, then there exists a $U^{\prime} \in \mathcal{U}^{\prime}$ such that $x \in \operatorname{cl}\left(U^{\prime}\right)$.

Example 2. Let $X=[0, \Omega)$ with the order topology. Every open cover of $X$ has an open order closure-preserving refinement, but $X$ is not paracompact. Let $v$ be an open cover of $X$, and let $x \in X$. If $x$ is a limit ordinal let $W_{x}$ be any open interval containing $x$ which is contained in some element of $\mathcal{V}$. If $x$ is an isolated point let $W_{x}=\{x\}$. Then $W=\left\{W_{x}: x \in[0, \Omega)\right\}$ is order closure-preserving with respect to the inverse of the usual order on the index set $[0, \Omega)$, and clearly an open refinement of $\mathcal{U}$.

It is possible, however, to get some results if we consider wellordered collections. Call a collection well-ordered closure-preserving if it is order closure-preserving with respect to a well-order. A collection $\mathcal{U}$ endowed with a well-order $\leqq$ is said to be well-ordered cushioned in a collection $\mathcal{V}$ with cushion map $f: \mathcal{U} \rightarrow \mathcal{V}$ provided for every $U \in \mathcal{U}$ and for every $x \in U$ and every subcollection $\mathcal{u}^{\prime} \subset\{V \in \mathcal{U}: V \leqq U\}$ if $x \in \operatorname{cl}\left(\cup u^{\prime}\right)$, then $x \in \bigcup f\left(\mathcal{u}^{\prime}\right)$. We can now incorporate Katuta's result in the following theorem.

Theorem 2. Let $X$ be a regular space. The following are equivalent.

(a) $X$ is paracompact.

(b) Every open cover of $X$ has an open refinement which is order locally finite. 
(c) Every open cover of $X$ has an open refinement which is wellordered closure-preserving.

(d) Every open cover of $X$ has an open refinement which is wellordered cushioned in it.

Proof. Katuta proved that (a) and (b) are equivalent. Clearly (a) $\Rightarrow(\mathrm{c}) \Rightarrow(\mathrm{d})$. The proof that $(\mathrm{d}) \Rightarrow(\mathrm{a})$ is similar to the proof that $(\mathrm{d}) \Rightarrow(\mathrm{a})$ in Theorem 1 .

The author would like to thank the referee for bringing Katuta's results to his attention, and for other helpful comments.

\section{REFERENCES}

1. J. Dieudonné, Une gênéralisation des espaces compacts, J. Math. Pures Appl. 23 (1944), 65-76. MR 7, 134.

2. Y. Katuta, $A$ theorem on paracompactness of product spaces, Proc. Japan Acad. 43 (1967), 615-618. MR 36 \#5894.

3. E. Michael, A note on paracompact spaces, Proc. Amer. Math. Soc. 4 (1953), 831-838. MR 15, 144.

4. - Another note on paracompact spaces, Proc. Amer. Math. Soc. 8 (1957), 822-828. MR 19, 299.

5. - Yet another note on paracompact spaces, Proc. Amer. Math. Soc. 10 (1959), 309-314. MR 21 \#4406.

6. - A note on closed maps and compact sets, Israel J. Math. 2 (1964), 173176. MR $31 \# 1659$.

7. H. Tamano, Note on paracompactness, J. Math. Kyoto Univ. 3 (1963), 137-143. MR 28 \#5419.

8. - On some characterizations of paracompactness, Topology Conference, Arizona State University, Tempe, Arizona, 1967, pp. 277-285.

University of North Carolina at Chapel Hill 